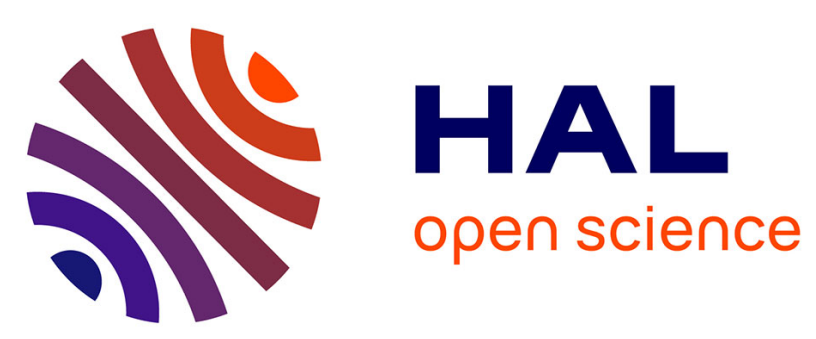

\title{
Patterns in magnetic granular media at the crossover from two to three dimensions
}

\author{
Eric Opsomer, Simon Merminod, Julien Schockmel, Nicolas Vandewalle, \\ Michael Berhanu, Eric Falcon
}

\section{- To cite this version:}

Eric Opsomer, Simon Merminod, Julien Schockmel, Nicolas Vandewalle, Michael Berhanu, et al.. Patterns in magnetic granular media at the crossover from two to three dimensions. Physical Review E , 2020, 102, pp.042907. 10.1103/PhysRevE.102.042907 . hal-02974898

\section{HAL Id: hal-02974898 \\ https://hal.science/hal-02974898}

Submitted on 22 Oct 2020

HAL is a multi-disciplinary open access archive for the deposit and dissemination of scientific research documents, whether they are published or not. The documents may come from teaching and research institutions in France or abroad, or from public or private research centers.
L'archive ouverte pluridisciplinaire HAL, est destinée au dépôt et à la diffusion de documents scientifiques de niveau recherche, publiés ou non, émanant des établissements d'enseignement et de recherche français ou étrangers, des laboratoires publics ou privés. 


\title{
Patterns in magnetic granular media at the crossover from two to three dimensions
}

\author{
Eric Opsomer $\odot,{ }^{1}$ Simon Merminod $\odot,{ }^{2,}{ }^{*}$ Julien Schockmel $\odot,{ }^{1}$ Nicolas Vandewalle, ${ }^{1}$ Michael Berhanu $\odot,{ }^{2}$ and Eric Falcon $\odot{ }^{2}$ \\ ${ }^{1}$ Université de Liège, GRASP, CESAM, B-4000 Liège, Belgium \\ ${ }^{2}$ Université de Paris, Université Paris Diderot, MSC, UMR7057 CNRS, F-75013 Paris, France
}

(Received 2 September 2020; accepted 4 October 2020; published 21 October 2020)

\begin{abstract}
We perform three-dimensional particle-based simulations of confined, vibrated, and magnetizable beads to study the effect of cell geometry on pattern selection. For quasi-two-dimensional systems, we reproduce previously observed macroscopic patterns such as hexagonal crystals and labyrinthine structures. For systems at the crossover from two to three dimensions, labyrinthine branches shorten and are replaced by triplets of beads forming upright triangles which self-organize into a herringbone pattern. This transition is associated with increases in both translational and orientational orders.
\end{abstract}

DOI: 10.1103/PhysRevE.102.042907

\section{INTRODUCTION}

Phase transitions result at the microscopic scale from the competition between thermal agitation and interactions between particles [1,2]. For finite-size systems, confinement plays an important role as well. Indeed, mechanical constraints can induce a large variety of structural phases, as it was shown for hard spheres confined between two parallel plates [3]. A frequently encountered consequence of such confinement is the emergence of buckling. The latter generally occurs when the state of minimal energy cannot be reached due to the limited available space in the system. Upon increasing confinement, structures reorganize. For example, parallel bands transform into chevrons. A regular array of organized chevrons is commonly called a herringbone pattern. Such pattern results from geometric frustrations due to confinement. Herringbone patterns are observed in various physical systems, such as ferroelectric materials [4], the crystal surface of metals [5], compressed elastic thin films [6,7], liquid crystals [8], magnetic garnet films [9], or ferrofluids [10,11], which are all continuous systems. In contrast, for particulate systems, the effect of confinement on pattern selection remains unexplored.

Counterparts of phase transitions can be observed in macroscopic systems such as vibrated granular media. Indeed, by adjusting the driving intensity, analogs of liquid-vapor and liquid-solid transitions occur in confined granular systems [12]. Due to the dissipation caused by particle collisions, a constant driving, injecting energy, is needed to ensure a stationary state, corresponding in this case to a nonequilibrium steady state [13]. This driving can be achieved mechanically [14-16] as well as magnetically [17,18]. To mimic phase transitions in molecular systems, where long-range correlations are possible, remote interactions must be introduced into granular systems. Liquid-solid-like transitions and self-organization occurring when the interaction strength

\footnotetext{
*Present address: Brandeis University, Martin A. Fisher School of Physics, Waltham, MA 02453, USA.
}

dominates over the agitation have been reported in numerous experiments using electrostatic $[19,20]$ or magnetic interactions [21-24]. A paradigm for granular media presenting remote interactions is given by mechanically agitated softferromagnetic beads enclosed in a quasi-two-dimensional cell. By applying an external magnetic field perpendicular to the cell, particles interact via two-body dipolar interactions of strength proportional to the square of the applied magnetic field. The interaction strength is thus a tunable parameter that can be externally controlled just like the driving amplitude and frequency of the vibrations applied to the cell. Depending on the number of particles inside the cell, experiments reported emergence of hexagonal crystal phases $[25,26]$ or disordered phases [27]. Despite the out-of-equilibrium character of vibrated granular systems, some phases obtained for large interaction strength are similar to the ones obtained in 2D colloidal systems [28,29] in which particles are large enough to be observed by microscope while being small enough to be thermally agitated. In particular, the experimental verification of the Kosterlitz-Thouless-Halperin-Nelson-Young (KTHNY) scenario in the melting of hexagonal crystals has been realized using magnetized colloids [30,31].

In addition to the number of particles enclosed within the cell, the magnitudes of the magnetic field and the agitation, the cell height has a strong influence on the structures that are observed in this kind of systems. Indeed, if almost no vertical motion is possible, the system remains two-dimensional, which yields strictly repulsive magnetic particle interactions. Accordingly, at high enough values of the applied magnetic field, when the potential magnetic energy dominates over the kinetic energy of particles, a crystalline structure minimizing the potential energy of the system is observed [Fig. 1(a)]. As one slightly increases the cell height, the structure of minimal energy becomes a buckled chain [Fig. 1(b)]. Due to their nonisotropic interaction potential, two particles in contact and partially overlapping attract each other, whereas particles far apart repel each other. Without confinement, particles would self-organize into vertical structures because gravity is negligible here. Thus, buckling results from the competition between the interactions and the confinement. The distribution 
(a)

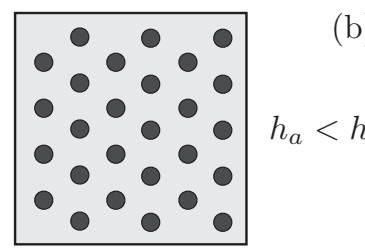

(b)
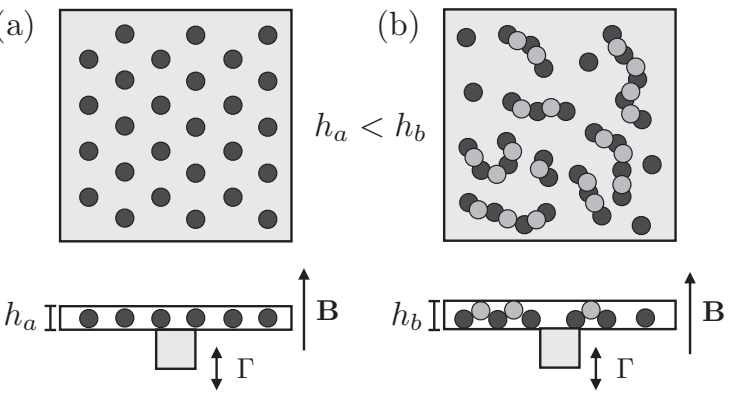

FIG. 1. (a) Top and (b) side views of a confined system of magnetic granular material. At fixed number of particles and magnetic field $\mathbf{B}$, decreasing the degree of confinement, i.e., the cell height, leads to the formation of labyrinthine patterns. $\Gamma$ denotes the dimensionless acceleration of the vibrations.

of particles in the horizontal plane follows, similar criteria based on energy minimization. Due to the complex energy landscape associated with the buckling, the system evolves to a disordered metastable state such as the tortuous chains in a labyrinthine phase [27].

In this article, we investigate the effect of larger cell heights for which the system is at the crossover from two to three dimensions. Since measuring the three-dimensional motions and positions remains challenging in experiments with overlapping particles, our study is realized using particle-based simulations. We accurately reproduce previous experimental results and explore several new regimes that are encountered as the cell height is increased. Finally, for quasi-three-dimensional systems, we report a structure displaying translational and orientational order in the form of a herringbone pattern.

\section{SETUP AND NUMERICAL MODEL}

Our work is inspired by previous experiments that we performed on the dynamics of confined granular gases with repulsive interactions [26]. The experimental system, that we reproduce numerically, consists of a quasi-two-dimensional aluminum cell with a bottom of side lengths $L_{x}$ and $L_{y}$ and a height $h$ (Fig. 2). Its bottom is covered with a sandpaper sheet to provide macroscopic roughness. Given the thickness $\xi$ of the sandpaper, the effective height of the system is reduced to $h-\xi$. The cell is filled with $N$ spherical chrome steel bearing beads of diameter $a=1 \mathrm{~mm}$ and closed by a smooth transparent polycarbonate lid. To obtain a granular gas, the cell is vibrated vertically according to a sinusoidal motion of frequency $f$ and dimensionless acceleration $\Gamma=A(2 \pi f)^{2} / g$, where $A$ is the driving amplitude and $g=9.81 \mathrm{~m} / \mathrm{s}^{2}$. Finally, to introduce remote interactions between the particles, the cell is placed in a magnetic field $\mathbf{B}$. Under such conditions, the soft-ferromagnetic particles behave as induced dipoles whose magnetic moments $\mathbf{m}$ are defined by

$$
\mathbf{m}=\frac{\pi a^{3}}{6} \frac{\chi_{m}}{\mu_{0}} \mathbf{B},
$$

where $\mu_{0}$ is the vacuum permeability and $\chi_{m}$ is the volume magnetic susceptibility. The latter was measured for the AISI 52100 chrome steel beads used in [26] and a value of 2.98 was
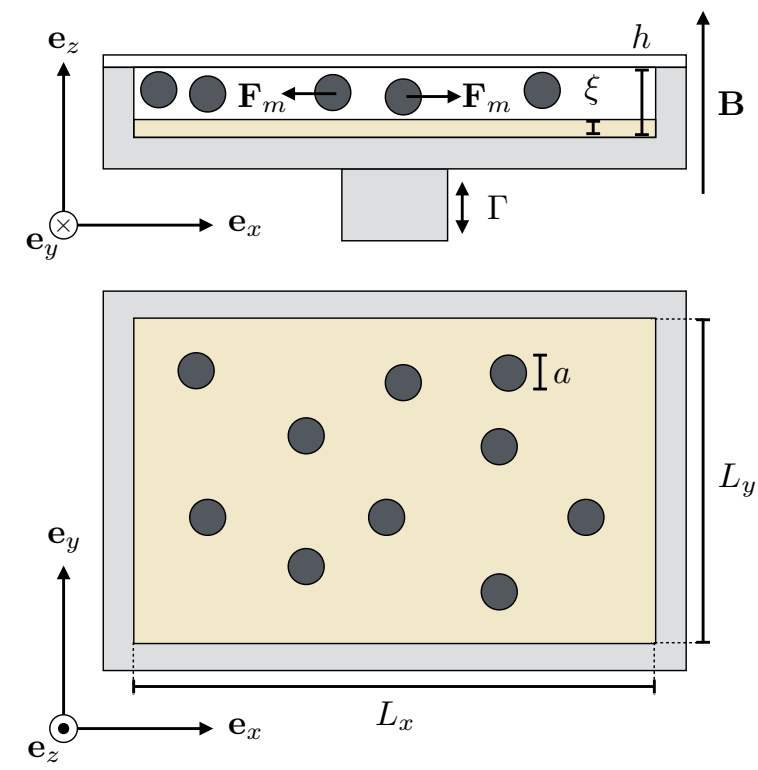

FIG. 2. Side and top view of the experimental setup that is reproduced numerically. An $L_{x} \times L_{y} \times h$ cell, enclosing $N$ spherical beads of diameter $a$, is vertically vibrated with a dimensionless acceleration $\Gamma$. The system is placed in a vertical magnetic field $\mathbf{B}$ which induces magnetic dipolar forces $\mathbf{F}_{m}$ between the particles.

obtained. Since a purely vertical magnetic field $\mathbf{B}=B \mathbf{e}_{z}$ was used, the magnetic force, exerted by one particle on another $\mathbf{F}_{m}$, is given by

$$
\mathbf{F}_{m}=\frac{3 \mu_{0} \mathbf{m}^{2}}{4 \pi|\mathbf{r}|^{4}}\left[\mathbf{n}+2\left(\mathbf{n} \cdot \mathbf{e}_{z}\right) \mathbf{e}_{z}-5\left(\mathbf{n} \cdot \mathbf{e}_{z}\right)^{2} \mathbf{n}\right],
$$

where $\mathbf{r}$ is the vector joining the centers of both particles, $\mathbf{n}=\mathbf{r} /|\mathbf{r}|$, and $\mathbf{e}_{z}$ is a vertical unit vector. More accurately, the magnetic dipoles in the particles are not only the result of the external magnetic field, but also of the induced fields of all particles. However, given the complexity of the resulting interactions, such nonlinear magnetization would be difficult to implement in our model. Fortunately, the amplitude of the magnetic field created by a neighboring bead never exceeds $B / 8$, as shown previously [32]. Therefore, our model enables us to qualitatively reproduce self-organization and phase transitions without taking into account the nonlinear magnetization of the beads. We now describe how to model such a system using numerical simulations.

\section{A. Particle-based simulations}

Our study is based on three-dimensional particle-based simulations [33-35] taking into account the possibility of strong overlaps between particles. Our custom-made algorithm operates with a constant time step and solves Newton's equations of motion using a leapfrog integration scheme. The different involved force models are described in the following.

For each collision between particles, the repulsive contact force $\mathbf{F}_{c}$ exerted by one particle on another can be decomposed into two components,

$$
\mathbf{F}_{c}=F_{n} \mathbf{n}+F_{t} \mathbf{t}
$$


where $F_{n}$ and $F_{t}$ are the normal and the tangential components, and $\mathbf{n}$ and $\mathbf{t}$ are two unit vectors pointing in the normal and tangential directions. Numerically, the deformation that occurs during this collision corresponds to an overlap $\delta=|\mathbf{r}|-a$ of the particles. The normal component $F_{n}$ depends on that overlap and is modeled using a linear spring coupled to a dashpot. One has

$$
F_{n}=-k_{n} \delta-\gamma_{n} \frac{d \delta}{d t},
$$

where the viscous constant $\gamma_{n}$ is a function of the particle stiffness $k_{n}$ and of the restitution coefficient $e$ characterizing the energy dissipation during the collision. The tangential component $F_{t}$ depends on the relative contact point velocity u and is bounded by Coulomb's law,

$$
F_{t}=-k_{t} \mathbf{u} \cdot \mathbf{t}, \quad\left|F_{t}\right| \leqslant \mu\left|F_{n}\right|,
$$

where $k_{t}$ is a large positive constant and $\mu$ is a dynamic friction coefficient. Contacts between particles and the walls are treated in a similar way. However, in the experiment, when a particle impacts the sandpaper, it will rebound erratically since a fraction of its vertical velocity is randomly redirected in the orthogonal directions. In the simulations, this can be modeled by a random reorientation of the normal vector at the lower surface respecting the following constraints:

$$
|\cos \alpha| \leqslant \mathbf{n} \cdot \mathbf{e}_{z}, \quad|\mathbf{n}|=1,
$$

where $\alpha$ is the maximum angle between $\mathbf{n}$ and the vertical direction. This angle has to be calibrated to reproduce the experimentally observed dynamics.

Since our particles act as magnetic dipoles, they are submitted to a magnetic force $\mathbf{F}_{m}$ as defined in Eq. (2). It is straightforward to add this force into the numerical model. However, to increase the computational speed, not all pairs of particles are treated and a cutoff distance of about ten particle diameters is introduced. Finally, the gravity force acting on each particle is taken into account.

\section{B. Parameter calibration}

To obtain realistic results in our simulations, the values of the different parameters of the model must be selected carefully. The normal stiffness $k_{n}=200 \mathrm{~N} / \mathrm{m}$ is chosen to ensure small overlaps (i.e., $|\delta| \leqslant a / 100$ ), even for the highest experimentally observed impact speeds. Tangential stiffness is fixed at $10^{6} \mathrm{~N} \mathrm{~s} / \mathrm{m}$. For simplicity, a unique friction coefficient $\mu=0.5$ is considered for all types of contact. For collisions between two particles as well as for collisions between a particle and a wall, a restitution coefficient $e_{g}=0.9$ (leading to $\gamma_{n}=4.19 \times 10^{-6} \mathrm{~Pa}$ s) is used. Since only limited data is available for them in literature, the restitution coefficient, characterizing the particle-sandpaper interaction, $e_{s}$ and the angle $\alpha$ are calibrated by comparing the particle velocity distributions in the simulation to experimental data. Measurements are realized in the setup described in Fig. 2 for $L_{x}=L_{y}=90 \mathrm{~mm}, h=1.5 \mathrm{~mm}$, and $N=1$, far away from the boundaries of the cell and without any magnetic field. As for all following simulations, the driving parameter are fixed to a frequency $f=300 \mathrm{~Hz}$ and a dimensionless acceleration $\Gamma=2.23$. The best agreement between
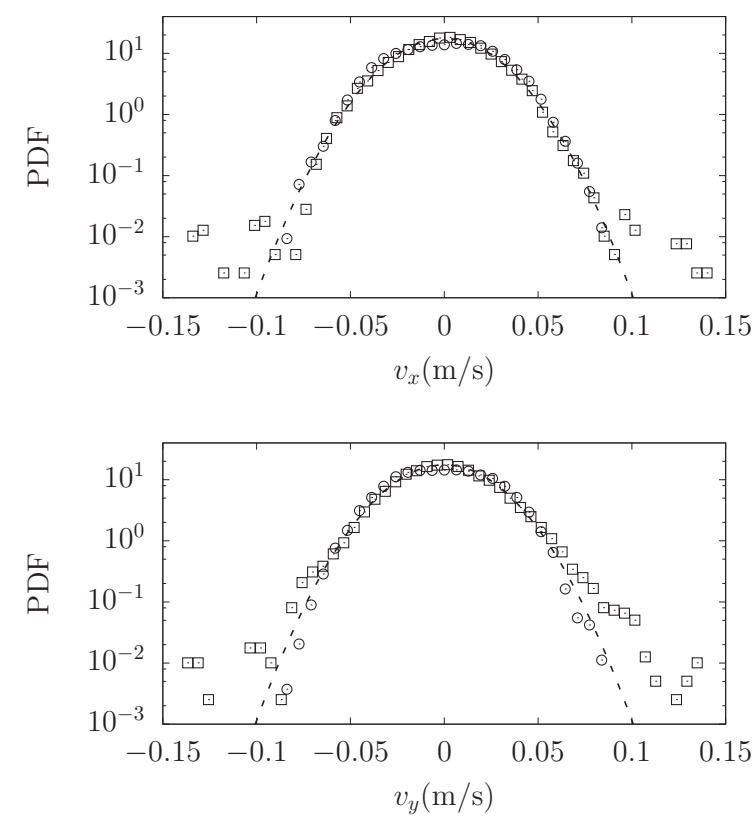

FIG. 3. Probability density function of $v_{x}$ (top) and $v_{y}$ (bottom), denoting, respectively, the horizontal velocities of the particle centers along the $x$ and $y$ directions of the system. Squares and circles correspond to experiments and simulations, respectively. Dashed lines correspond to a Gaussian fits of the experimental data.

experiment and simulations is obtained for $e_{s}=0.7$ and $\alpha=0.044$. Figure 3 shows the probability density functions (PDFs) of $v_{x}$ and $v_{y}$, which denote, respectively, the horizontal velocities of the particle centers along the $x$ and $y$ directions of the system. Experimental data are represented by squares while numerical data are represented by circles. The dashed lines correspond to a Gaussian fit of the experimental data. Note that our calibration takes into account the thickness $\xi$ of the sandpaper at the bottom of the experimental cell which was estimated to be approximatively $85 \mu \mathrm{m}$.

To validate our numerical model, we reproduce our previous experimental results [26]. For this, we run simulations in which $N=2000$ particles are placed in the same container, which leads to a area fraction $\phi=0.194$. The magnetic field is switched on and the system is vibrated during $10 \mathrm{~s}$ using the driving parameters detailed in the calibration section. As shown previously [26], the dynamics of the experimental system are controlled by the ratio $\varepsilon=E_{m} / E_{k}$ comparing the magnetic potential energy to the kinetic energy in the horizontal plane. In our numerical system, these energies are defined as follows:

$$
\begin{gathered}
E_{m}=\frac{\pi a^{6}}{16 \mu_{0}} B^{2} \overline{\left\langle\left(r_{x}^{2}+r_{y}^{2}\right)^{-3 / 2}\right\rangle}, \\
E_{k}=\frac{1}{2} m \overline{\left\langle v_{x}^{2}+v_{y}^{2}\right\rangle} .
\end{gathered}
$$

where $\langle\cdot\rangle$ denotes the average over all particles in the central region of the cell and ${ }^{-}$denotes the average over the last $2 \mathrm{~s}$ of the simulation. Figure 4 shows snapshots $(35 \mathrm{~mm} \times 35 \mathrm{~mm})$ of the system for different values of $\varepsilon$. Experiments [26] are presented in the top row and simulations in the bottom row. For small $\varepsilon$ values, the system behaves as a collisional gas 


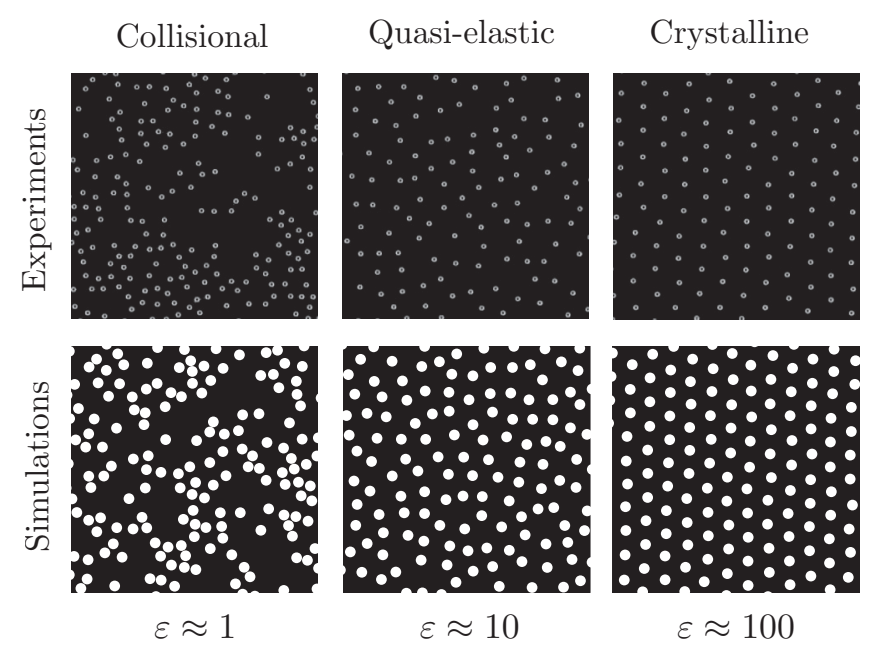

FIG. 4. Snapshots of dilute systems for different values of the energy ratio $\varepsilon=E_{m} / E_{k}$. Top row shows our pevious experimental results (adapted from Ref. [26]). Bottom row shows our simulations. Different dynamical regimes are reproduced (see text) and an excellent qualitative agreement is found between experiments and simulations for the particle's structural arrangements.

and clustering is observed. For intermediate $\varepsilon$, most collisions between grains are avoided and the system enters a quasielastic regime. For high $\varepsilon$, the dynamics are dominated by the magnetic repulsion and the grains self-organize into a crystalline structure. Excellent qualitative agreement is found between our simulations and the experimental data. The particles seems smaller in the experiments because the observable bright spots are smaller than $a$.

\section{RESULTS}

According to earlier works [27,28], one expects the apparition of labyrinthine structures for high fields and high surface fraction. Indeed, under these circumstances, the granular material self-organizes into chains whose constitutive particles touch alternatively the bottom and the top wall of the container [as illustrated in Fig. 1(b)]. This structure is energetically more favorable than any two dimensional crystalline arrangement. Since buckling involves the third dimension of the system, we decided to numerically investigate the effect of the cell height on the observed structures. Using the same calibration as above, we realized simulations for $N=2291$ grains placed in a container of side length $L_{x}=L_{y}=60 \mathrm{~mm}$ which leads to $\phi=0.5$. Several values of $h$ varying in between $1.2 \mathrm{~mm}$ and $2.0 \mathrm{~mm}$ are tested. Once the driving starts, the magnetic field strength is increased linearly during 10 seconds until a value of $B=300 \mathrm{G}(\varepsilon \gg 100)$ is reached. The rate of the magnetic ramp, in our case $\tau=30 \mathrm{G} / \mathrm{s}$, does not impact the local structures near the contacts but rather affects the characteristics of the disorder on a larger scale (chain length, chain orientation, branching). Thus, changing this rate does not modify the type of phase that is observed but only changes some of its characteristics, as also reported in Ref. [32]. Simulations are ended after another ten seconds of driving under constant magnetic field conditions.

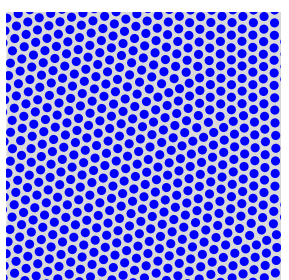

$$
h / a=1.2
$$
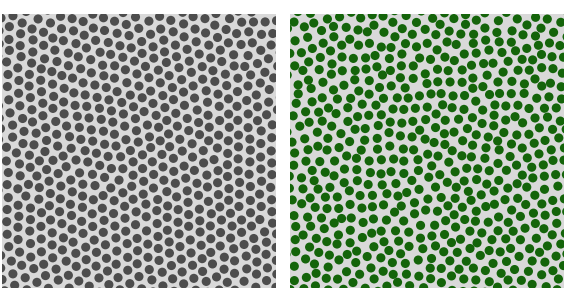

$h / a=1.3$

$h / a=1.4$

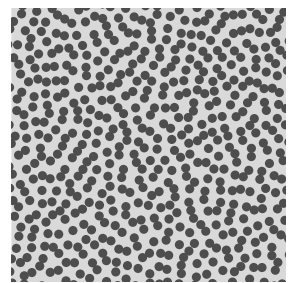

$h / a=1.5$

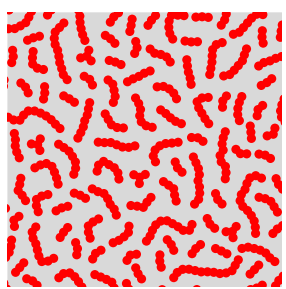

$h / a=1.8$

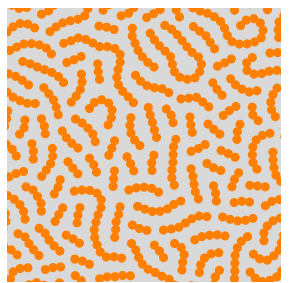

$h / a=1.6$

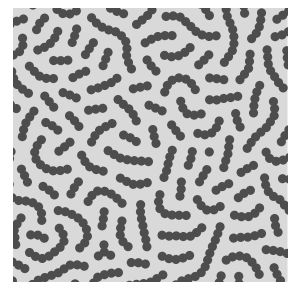

$h / a=1.7$
FIG. 5. Snapshots $(30 \mathrm{~mm} \times 30 \mathrm{~mm})$ of the numerical system for selected cell height values $h / a$. A variety structures are observed as the height is increased: hexagonal crystals, disordered fluid, long chains and labyrinthine structures, small individual chains and upright triangles. Colors correspond to $h / a$ values that are also used in other figures.

An overview of the different final states is given in Fig. 5 presenting snapshots $(30 \mathrm{~mm} \times 30 \mathrm{~mm})$ of the system for selected $h / a$ values. As expected, the increase of the cell height $h$ leads to consecutive structural transitions from a crystalline arrangement toward disordered individual grains that eventually gather into long chains of several particles and form labyrinthine patterns. Similar structures were reported previously both experimentally for paramagnetic grains and colloids [27-29] and numerically for systems with particles interacting by a combination of long-range repulsion and short-range attraction [36,37], a combination of hard- and soft-core repulsion [38], or dipolar interactions [29,39].

As $h$ continues to grow the buckling of the chains intensifies, which means that the vertical distances $\Delta z$ between first neighbors increase while the horizontal distances $\Delta \rho$ between them decrease so that, seen from above, the chains seem to shorten. Above a critical cell height, another structural transition occurs: chains vanish and grains start to gather three by three in upright triangular structures. Geometrically, this becomes possible once $h / a>\xi / a+1+\sqrt{3} / 2 \approx 1.95$ which is in good agreement with Fig. 5. Although similar structures, made of two overlapping beads, were reported by Dobnikar et al. [39], it is, to the best of our knowledge, the first time that such triangular structures are reported in a macroscopic particulate system. This could be related to the fact that only 


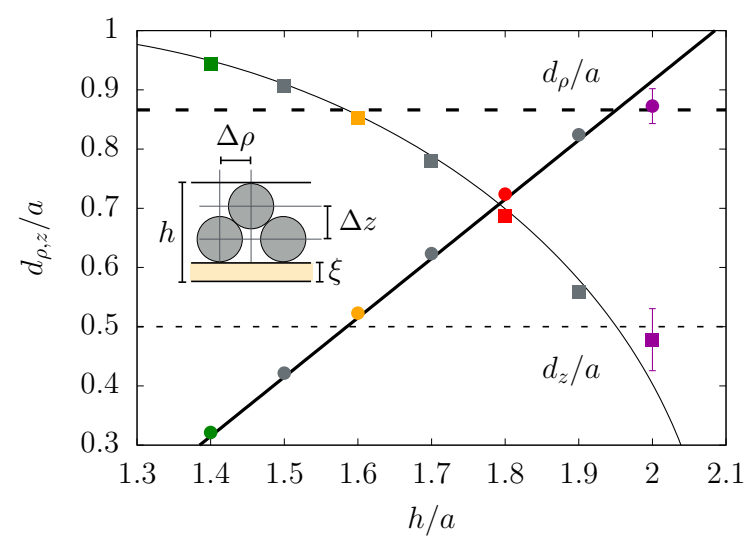

FIG. 6. Evolution of the average vertical and horizontal distances $d_{z} / a$ and $d_{\rho} / a$ between first neighbors for growing cell height, $h / a$. Measurements are represented, respectively, by bullets and squares. Error bars of the data points correspond to the measured standard deviations and are often smaller than the symbols. Theoretical predictions of $d_{z} / a$ and $d_{\rho} / a$, based on the geometry of isosceles triangles, are represented, respectively, by thick and thin curves. Among them, solid curves correspond to constrained triangles while dashed curves correspond to constraint-free triangles.

few numerical studies take into account all three dimensions of the system.

To evaluate the impact of the confinement on buckling, we measure the average distances between first neighbors for all different cell heights. Results are shown in Fig. 6. The observed evolutions of $d_{z}=\overline{\langle\Delta z\rangle}$ and $d_{\rho}=\overline{\langle\Delta \rho\rangle}$, represented, respectively, by bullets and squares, can be predicted by considering the case where first neighbors touch alternatively the sandpaper and the lid of the container. For this configuration, one can define a right-triangle were $\Delta z$ and $\Delta \rho$ are the lengths of the legs and $a$ the length of the hypotenuse. By taking into account the cell height $h$ and the thickness $\xi$ of the sandpaper, one obtains

$$
\begin{gathered}
d_{z} / a=h / a-\xi / a-1, \\
d_{\rho} / a=\sqrt{1-\left(d_{z} / a\right)^{2}} .
\end{gathered}
$$

In Fig. 6, these relationships are represented by thick and thin solid black curves, respectively. Moreover, dashed horizontal lines correspond to the typical distances that are expected for a constraint-free equilateral triangle. As long as $h / a<1.95$, all data points follow the solid lines which means that the buckling is entirely controlled by $h$. Above this threshold, the structures in the system become independent of $h$ and data points lie on the dashed lines.

Another relevant parameter, giving an insight on the morphology of the system, is the average number of particles in a chain, denoted $N_{c}$. As shown in Fig. 7, this average chain length has a nonmonotonic evolution. Starting at $N_{c}=1$, the average chain length increases as soon as crystalline order is lost. A maximum of about five particles is reached during the labyrinthine regime. From there on, $N_{c}$ decreases toward 3 , which corresponds to the emergence of triangular structures. Note that measurements of particle positions would

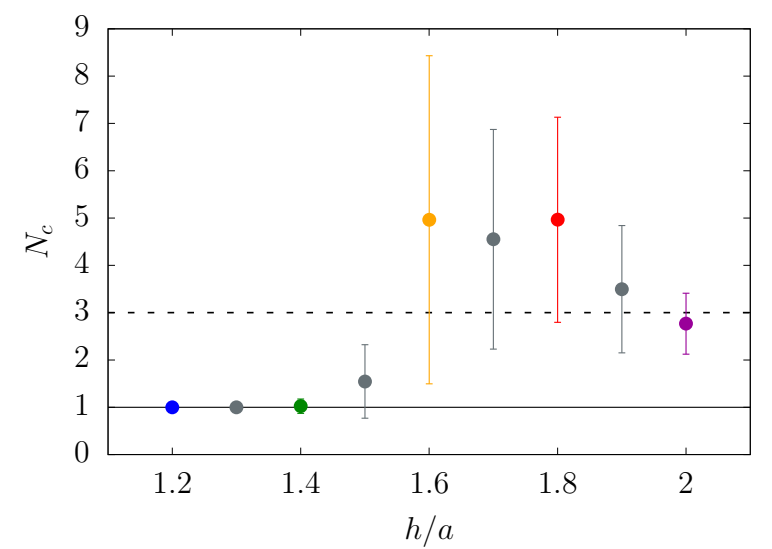

FIG. 7. Evolution of the average chain length $N_{c}$ as a function of the cell height, $h / a$. Error bars of the data points correspond to the measured standard deviations. The solid and dashed lines at $N_{c}=1$ and $N_{c}=3$ correspond, respectively, to the theoretical values expected for crystalline arrangements and triangular structures.

not always be possible experimentally because the important overlaps between particles prevent tracking them individually.

\section{STRUCTURAL ANALYSIS}

The important structural changes that appear in the system while modifying the degree of confinement can be characterized using the radial pair correlation function $g(\rho)$, which describes how density varies as a function of the horizontal distance $\rho$ from a reference particle. Figure 8 shows $g(\rho)$ for the investigated cell heights. Errorbars, corresponding to standard deviation, are given by a light envelope around each curve. To improve readability of the data, measurements are vertically shifted, each by four units. Dashed lines correspond therefore to zero values of each correlation function. Given the important overlaps between particles, this measurement could not have been realized experimentally. Confinement decreases from the top to the bottom. For $h / a=1.2$, one observes several peaks corresponding to the typical distances within a hexagonal lattice. At $h / a=1.4$, the system is disordered and displays no long range translational order. For $h / a=1.6$ and $h / a=1.8$, one can see several peaks corresponding to neighbors of a same chain each separated by an average distance $d_{\rho}$. As $h$ grows and buckling intensifies, these peaks tighten and shift leftward. The first peaks are about four times higher than the second peaks. This particular feature is explained by the presence of numerous small chains in the systems. After the second peaks, $g(\rho)$ always presents nonzero values due to nonaligned neighbors and grains from other chains. For $\rho / a \geqslant 3$, no more correlations are observed. For $h / a=2.0$, the purple $g(\rho)$ function shows peaks at $\rho / a=0.5$ and $\rho / a=$ 1 corresponding to particles making up the same triangles. In the region $1.5 \leqslant \rho / a \leqslant 3$, three nonresolved peaks correspond to first neighbor triangles. Much alike more confined systems, correlation disappears beyond a distance of about $3 a$. The gray curve (labeled "top") is obtained by computing $g(\rho)$ while considering only the top particles of each triangular structure. The first gray peak, corresponding to the average distance between two neighboring structures, is detected at 


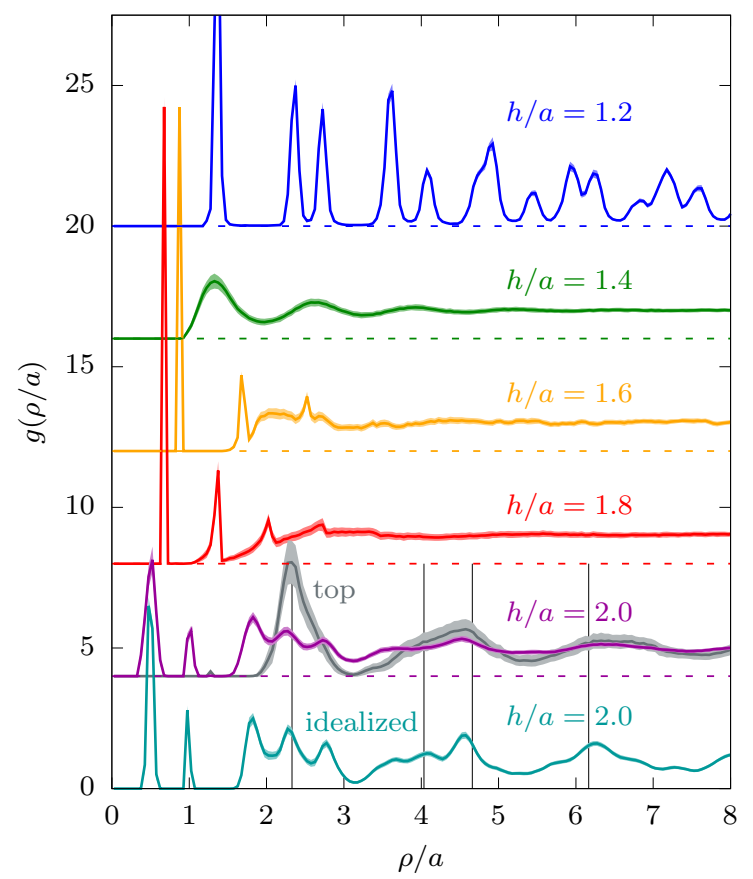

FIG. 8. Radial pair correlation function $g(\rho)$ of the horizontal particle distances $\rho$ for different cell heights $1.2 \leqslant h / a \leqslant 2.0$ (solid curves). For clarity, curves are vertically shifted, each by four units. Light envelopes around each curve correspond to errorbars. The teal curve is obtained for on idealized systems with $h / a=2.0$. The gray curve is obtained by considering only the top particles of the triangular structures in a regular system. The dashed lines show the zero value of each correlation function. The vertical lines correspond to the characteristic distances in a hexagonal lattice.

$\rho / a \approx 2.33$. For $\rho / a>2.33$, additional unresolved peaks can be observed near the characteristic distances of a hexagonal lattice (vertical lines in Fig. 8). One can verify this hypothesis by assuming that the top particle of each triangle lies on a vertex of a hexagonal lattice of mesh size $s$. In that case, equaling the surface fraction of one mesh in the upper layer to $\phi / 3$ yields the following relation:

$$
\frac{\pi a^{2} / 8}{\sqrt{3} s^{2} / 4}=\frac{\phi}{3} \Leftrightarrow s / a=\sqrt{\frac{\sqrt{3} \pi}{2 \phi}}=2.33 .
$$

This particular crystalline arrangement is a clear trace of translational order within the system and will be the starting point for the conception of an idealized system.

\section{HERRINGBONE PATTERNS}

Since the top grain of each triangle was found located at a vertex of an hexagonal lattice, we expected orientational order to arise among the short chains as well. However, this was not the case as one can see in Fig. 5. To promote orientational order, we realize another series of simulations using an environment which is more adapted to the crystalline structure of our system and that we call "idealized system": (i) During initialization, grains are deliberately placed in triangular arrangements. To stabilize these structures, the magnetic field is directly set to $300 \mathrm{G}$. (ii) Triangles are placed on the vertices of a hexagonal lattice whose mesh size $s$ is commensurable

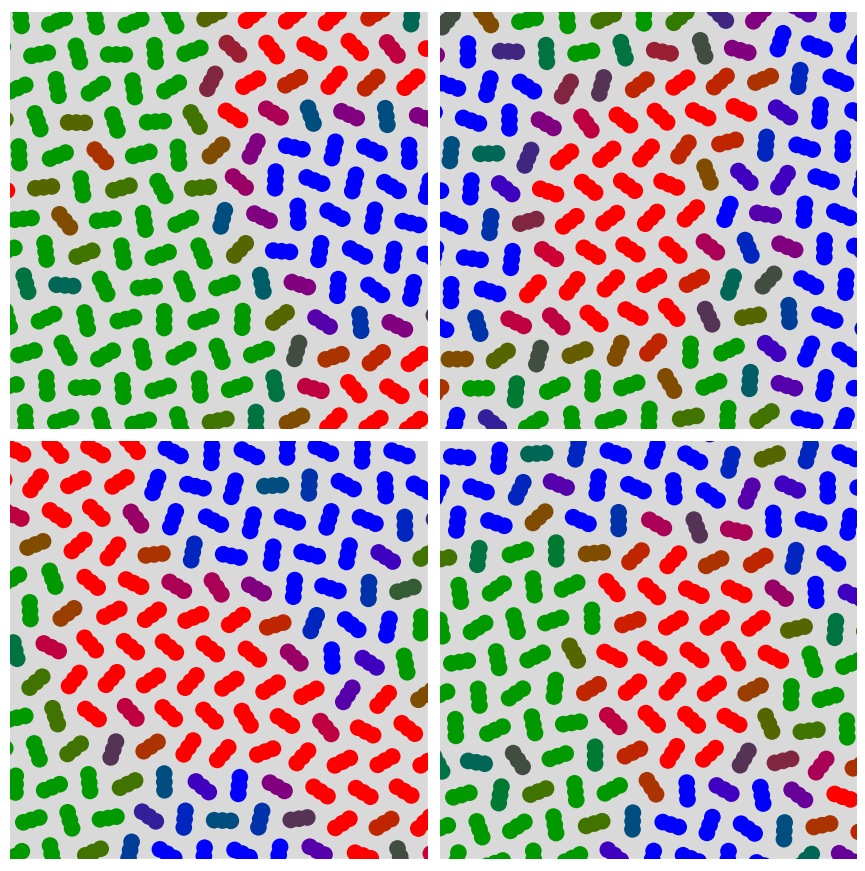

FIG. 9. Snapshots $(25 \mathrm{~mm} \times 25 \mathrm{~mm})$ of four idealized systems of dimensions $L_{x}=32.6 \mathrm{~mm}, L_{y}=32.3 \mathrm{~mm}$ and $h=2.0 \mathrm{~mm}$ and filled with $N=672$ particles. Vertical triangular structures arise and arrange into several domains presenting a herringbone pattern in the horizontal plane. Particles are colored with regards to the director field of their domain.

with the dimensions of the system. Note that after the positioning, all structures are free to move during the simulation. (iii) Periodic boundary conditions are implemented to decrease the number of defects along the lateral cell walls. We emphasize, that the emergence of the triangular structures is not a consequence of these particular initial conditions. Our idealized system allows merely for a faster relaxation of the system toward a configuration of least energy. To comply with (i) and (ii), $N$ must be a multiple of 3 and the dimensions of the container must scale as $L_{x}=n_{x} s$ and $L_{y}=n_{y} \sqrt{3} s, n_{x}$ and $n_{y}$ being integers. The number of grains is then given by the following formula:

$$
N=3\left\lfloor\frac{4 \phi n_{x} n_{y} \sqrt{3}}{3 \pi}(s / a)^{2}\right\rfloor,
$$

where $\lfloor\cdot 7$ denotes the nearest integer function. By keeping $\phi \approx 0.5$ and choosing $n_{x}=14$ and $n_{y}=8$, we obtain a container of dimensions $L_{x}=32.6 \mathrm{~mm}, L_{y}=32.3 \mathrm{~mm}$, and $h=2.0 \mathrm{~mm}$, filled with $N=672$ particles. Several simulations were realized in this idealized system, using the usual calibration and driving parameters. Figure 9 shows four snapshots $(25 \mathrm{~mm} \times 25 \mathrm{~mm})$, obtained after $10 \mathrm{~s}$ of driving. Qualitatively, one can see that this idealized system is much more organized than a regular one as show in Fig. 5. Several domains presenting herringbone patterns are observed and can be highlighted using different colors (details in the next section). Moreover, computing the radial pair correlation function for an idealized system leads to the solid teal curve (labeled "idealized") at the bottom of Fig. 8. As with the regular system, one observes peaks corresponding to particles sharing 


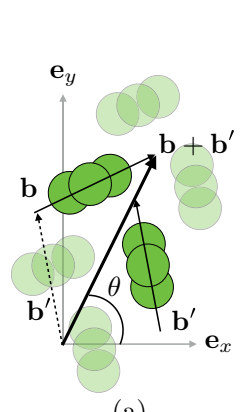

(a)

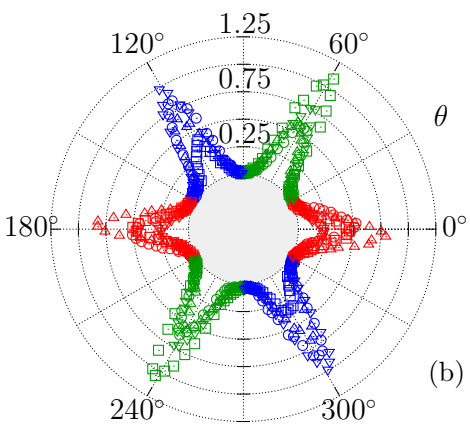

(b)

FIG. 10. (a) Geometric construction yielding the angle $\theta$. The norms of the vectors $\mathbf{b}$ and $\mathbf{b}^{\prime}$, associated to two neighboring triangles, are exaggerated to clarify the sketch. (b) Distribution of the angle $\theta$ characterizing the orientation of each wedge. Six peaks corresponding roughly to multiples of $60^{\circ}$ are observed. Symbols refer to four different simulations and are colored with respect to the corresponding herringbone domains as labeled in Fig. 9. Data is cumulated over the last second of the simulations.

the same triangular structure and to first neighbor triangles. However, for $\rho / a \geqslant 3$, the idealized case shows additional peaks corresponding to second and third neighbor triangles which indicates correlation at much longer range.

In the following, we discuss the orientational order that is observed in idealized systems. The elementary unit of a herringbone domain is made of two neighbor triangles that form a wedge of roughly 75 . Within a same domain, all wedges tend to align (or antialign) with a common director field. Each wedge can thus be associated to a particular domain (red, green or blue) based on its orientation. To determine this orientation, we define for each triangle a vector $\mathbf{b}$ joining the centers of its bottom grains. If two neighbor triangles form a wedge, their vectors $\mathbf{b}$ and $\mathbf{b}^{\prime}$ are chosen to point to one another. As shown in Fig. 10(a), summing these vectors leads to a resultant vector forming an angle $\theta$ with $\mathbf{e}_{x}$. Figure 10(b) shows the distribution of this angle using a polar plot. The radial component corresponds to the density of observation of the angular component $\theta$. Six peaks centered roughly around multiples of $60^{\circ}$ are observed and correspond to the orientation of the director fields of the different herringbone domains. Each wedge can thus be associate to one type of domain by determining to which peak its orientation angle $\theta$ belongs. Thanks to the distributions presented in Fig. 10, we can highlight pattern formation and emergence of local orientational order in the idealized numerical system. No global order is observed since several domains coexist and create defects along their frontiers. To validate our predictions, we tried to obtain the herringbone pattern experimentally. In spite of boundary effects and incomplete triangular structures, small herringbone domains could be observed (Fig. 11). Both snapshots $(35 \mathrm{~mm} \times 35 \mathrm{~mm})$ correspond to experiments realized in the original setup [26] with a packing fraction $\phi=0.5$, a magnetic field $B=400 \mathrm{G}$ and a dimensionless driving acceleration $\Gamma=1.6$ (which is weaker than in the rest of this article). The blue and green ovals show domains detected by the naked eye.

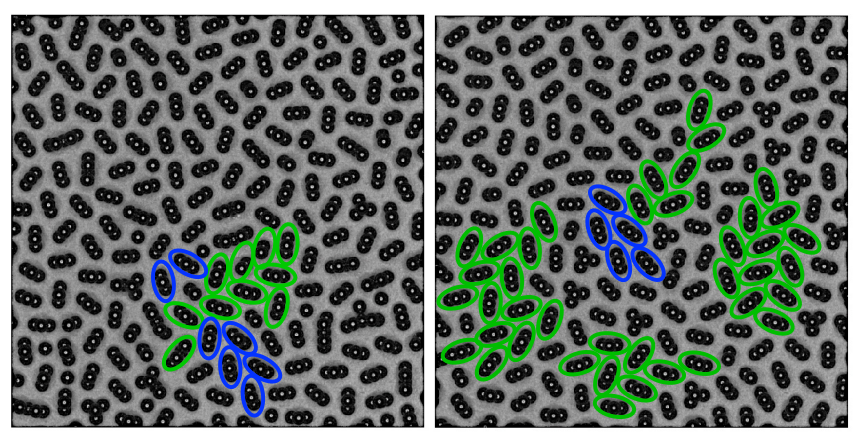

FIG. 11. Snapshots $(35 \mathrm{~mm} \times 35 \mathrm{~mm})$ of two experiments realized in the original setup with a packing fraction $\phi=0.5$, a magnetic field $B=400 \mathrm{G}$ and a dimensionless driving acceleration $\Gamma=1.6$. Domains are detected by the naked eye.

\section{CONCLUSION}

Using three-dimensional particle-based simulations, we reproduced previous experiments characterizing the formation of labyrinthine structures within a magnetized and vibrated granular medium. Since this phenomenon relied on the buckling of granular chains in the system, we explored the effect of the confinement on the observed structures. Granular chains and labyrinthine patterns remain present as long as a critical cell height is not exceeded. Above this threshold, new structures emerge: Particles in the system gather three by three to form upright triangles. The top grains of these triangles self-organize onto a hexagonal lattice in the dilute upper layer of the system. The bottom grains of neighboring triangles reorientate themselves to minimize the interaction energy. Two such triangles alone, would become parallel. However, for an assembly of multiple triangles, such arrangement is not compatible with the geometry of the upper layer crystal and the resulting frustration yields the emergence of a herringbone pattern. Due to defects, the system might be composed of several competing herringbone domains. By idealizing the initial conditions of our simulations, larger domains presenting clear translational and orientational order were observed. This work highlights the essential role of dimensionality and confinement in systems where complex pattern arise from competing mechanisms.

Even tough our numerical results were confirmed by first experiments, a thorough study with a hexagonal experimental cell should be realized in the future. Moreover, some theoretical argument predicting the particular angle in the herringbone pattern has still to be established.

\section{ACKNOWLEDGMENTS}

E.O. thanks Belspo-PRODEX. We thank European Space Agency (4000103461CCN) for financial support. The authors thank M. Noirhomme for complementary simulations and S. Dorbolo as well as M. Poty for fruitful discussions. 
[1] P. Chaikin and T. Lubensky, Principles of Condensed Matter Physics (Cambridge University Press, Cambridge, UK, 1995).

[2] J.-L. Barrat and J.-P. Hansen, Basic Concepts for Simple and Complex Liquids (Cambridge University Press, Cambridge, UK, 2003).

[3] M. Schmidt and H. Löwen, Phys. Rev. E 55, 7228 (1997).

[4] N. Choudhury, L. Walizer, S. Lisenkov, and L. Bellaiche, Nature 470, 513 (2011).

[5] S. Narasimhan and D. Vanderbilt, Phys. Rev. Lett. 69, 2455 (1992).

[6] X. Chen and J. W. Hutchinson, Scr. Mater. 50, 797 (2004).

[7] B. Audoly and A. Badaoud, J. Mech. Phys. Solids 56, 2401 (2008).

[8] J.-H. Huh, Y. Hidaka, A. G. Rossberg, and S. Kai, Phys. Rev. E 61, 2769 (2000).

[9] M. Seul and R. Wolfe, Phys. Rev. A 46, 7519 (1992).

[10] C. Flament, J.-C. Bacri, A. Cebers, F. Elias, and R. Perzynski, Europhys. Lett. 34, 225 (1996).

[11] F. Elias, C. Flament, J.-C. Bacri, and S. Neveu, J. Phys. I France 7, 711 (1997).

[12] N. Mujica and R. Soto, in Recent Advances in Fluid Dynamics with Environmental Applications (Springer, Berlin, 2016), pp. 445-463.

[13] B. Derrida, J. Stat. Mech. (2007) P07023.

[14] J. P. D. Clewett, J. Wade, R. M. Bowley, S. Herminghaus, M. R. Swift, and M. G. Mazza, Sci. Rep. 6, 28726 (2016).

[15] S. Aumaître, R. P. Behringer, A. Cazaubiel, E. Clément, J. Crassous, D. J. Durian, E. Falcon, S. Fauve, D. Fischer, A. Garcimartín, Y. Garrabos, M. Hou, X. Jia, C. Lecoutre, S. Luding, D. Maza, M. Noirhomme, E. Opsomer, F. Palencia, T. Pöschel, J. Schockmel, M. Sperl, R. Stannarius, N. Vandewalle, and P. Yu, Rev. Sci. Instrum. 89, 075103 (2018).

[16] A. Sack, K. Windows-Yule, M. Heckel, D. Werner, and T. Pöschel, Granular Matter 22, 54 (2020).

[17] E. Falcon, J.-C. Bacri, and C. Laroche, Europhys. Lett. 103, 64004 (2013).

[18] M. Adachi, P. Yu, and M. Sperl, npj Microgravity 5, 19 (2019).
[19] M. Saint-Jean, C. Guthmann, and G. Coupier, Eur. Phys. J. B 39, 61 (2004).

[20] I. S. Aranson, D. Blair, V. A. Kalatsky, G. W. Crabtree, W. K. Kwok, V. M. Vinokur, and U. Welp, Phys. Rev. Lett. 84, 3306 (2000).

[21] D. L. Blair and A. Kudrolli, Phys. Rev. E 67, 021302 (2003).

[22] D. Lopez and F. Pétrélis, Phys. Rev. Lett. 104, 158001 (2010).

[23] C. Laroche and F. Pétrélis, Eur. Phys. J. B 77, 489 (2010).

[24] L. Oyarte, P. Gutiérrez, S. Aumaître, and N. Mujica, Phys. Rev. E. 87, 022204 (2013).

[25] J. Schockmel, E. Mersch, N. Vandewalle, and G. Lumay, Phys. Rev. E. 87, 062201 (2013).

[26] S. Merminod, M. Berhanu, and E. Falcon, Europhys. Lett. 106, 44005 (2014).

[27] S. Merminod, T. Jamin, E. Falcon, and M. Berhanu, Phys. Rev. E 92, 062205 (2015)

[28] N. Osterman, D. Babic, I. Poberaj, J. Dobnikar, and P. Ziherl, Phys. Rev. Lett. 99, 248301 (2007).

[29] R. Messina, S. Aljamhari, L. Bécu, J. Schockmel, G. Lumay, and N. Vandewalle, Sci. Rep. 5, 10348 (2015).

[30] K. Zahn, R. Lenke, and G. Maret, Phys. Rev. Lett. 82, 2721 (1999).

[31] U. Gasser, C. Eisenmann, G. Maret, and P. Keim, ChemPhysChem 11, 963 (2010).

[32] S. Merminod, Self-organization of vibrated and magnetized particles: Structure, dynamics and transitions, Ph.D. thesis, University Paris Diderot, 2016. Available at http://www.msc.univparis-diderot.fr/ falcon/TheseMerminodFinal08042017.pdf.

[33] T. Pöschel and T. Schwager, Computational Granular Dynamics (Springer Verlag, Berlin/Heidelberg, 2005).

[34] F. Radjaï and F. Dubois, Discrete-element Modeling of Granular Materials (Wiley-ISTE, New York, 2011).

[35] M. P. Allen and D. J. Tildesley, Computer Simulations of Liquids (Oxford University Press, Oxford, UK, 2017).

[36] M. D. Haw, Phys. Rev. E 81, 031402 (2010).

[37] M. D. Haw, Phys. Rev. E 99, 012603 (2019).

[38] G. Malescio and G. Pellicane, Nature Mater. 2, 97 (2013).

[39] J. Dobnikar, J. Fornleitner, and G. Kahl, J. Phys.: Condens. Matter 20, 494220 (2008). 